\title{
DENSIDAD Y DISTANCIA DE SIEMBRA EN DOS VARIEDADES DE SOYA DE TEMPORAL EN VERACRUZ, MÉXICO ${ }^{1}$
}

\author{
Oscar Hugo Tosquy-Valle ${ }^{2}$,Valentín Alberto Esqueda-Esquivel ${ }^{2}$, Rigoberto Zetina-Lezama ${ }^{2}$, \\ Guillermo Ascencio-Luciano ${ }^{3}$
}

\begin{abstract}
RESUMEN
Densidad y distancia de siembra en dos variedades de soya de temporal en Veracruz, México. En Veracruz, México, con el objeto de determinar el efecto de distintas densidades de siembra en el comportamiento de variedades de soya. Se evaluaron tres distanciamientos entre surcos: 40, 60 y $75 \mathrm{~cm}$, tres densidades de siembra: 200000,300000 y 400000 plantas/ha y dos variedades: Huasteca 100 y Huasteca 200, en bloques al azar con cuatro repeticiones, en factorial 3 x 3 x 2 y arreglo de surcos en franjas, durante el temporal de 2002 y 2003. Se midieron: días a floración, altura de la planta, altura de la vaina baja, número de entrenudos, número de vainas por planta y rendimiento de semilla. En siembras tardías, con las distancias a 40 y $60 \mathrm{~cm}$, la altura de vaina baja fue significativamente mayor y se tuvieron más entrenudos, que a $75 \mathrm{~cm}$. A partir de 300000 plantas/ha, Huasteca 200, presentó una altura de vaina baja, superior a la que tuvo con la densidad menor. En siembras tempranas, los mayores rendimientos se obtuvieron con 300000 y 400000 plantas/ha. Huasteca 200 fue la variedad más tardía y de mayor altura de planta y de vaina baja, pero Huasteca 100 fue más rendidora. La mayor producción se obtuvo con Huasteca 100, a partir de 300000 plantas/ha.
\end{abstract}

Palabras clave: Glycine max, genotipos, distanciamiento entre surcos, población de plantas, comportamiento agronómico.

\begin{abstract}
Density and sowing distance for two rain-fed soybean varieties in Veracruz, Mexico. In Veracruz, Mexico, in order to determine the effect of different sowing densities on the behavior of soybean varieties, three row spacing distances: 40, 60 and $75 \mathrm{~cm}$, three plant densities: 200.000, 300.000 and 400000 plants/ha and two varieties: Huasteca 100 and Huasteca 200 were evaluated using a completely randomized block design with four replications, in a $3 \times 3$ x 2 factorial and rows arranged in stripes, during the rainy seasons of 2002 and 2003. Days to flowering, plant height, lower pod height, number of internodes and pods per plant and seed yield were measured. In late sowings, at 40 and $60 \mathrm{~cm}$ row spacing, the lower pod height was significantly higher and more internodes per plant were produced, than at $75 \mathrm{~cm}$ rows. From 300.000 plants/ha on, Huasteca 200 had a lower pod height superior than that obtained at the lowest density. In early sowings, the highest grain yields were produced with 300.000 and 400.000 plants/ha. Huasteca 200 was the latest variety and had higher plant and lower pod heights, but Huasteca 100 was more productive. The highest yield was obtained with Huasteca 100 starting at 300.000 plants/ha.
\end{abstract}

Key words: Glycine max, genotypes, row width, plant population, agronomic behavior.

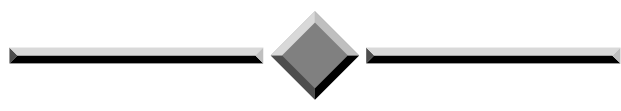

\footnotetext{
Recibido: 24 de marzo, 2009. Aceptado: 17 de mayo, 2010. Proyecto del Programa Nacional de Investigación de Soya del Instituto Nacional de Investigaciones Agrícolas y Pecuarias (INIFAP). Etapa 2002-2003. PRECI No. 2369.

2 Campo Experimental Cotaxtla. Instituto Nacional de Investigaciones Agrícolas y Pecuarias (INIFAP). Km 34 carr. Veracruz-Córdoba. Apdo. Postal 429, 91700, Veracruz, Ver., México. tosquy.oscar@inifap.gob.mx; esqueda.valentin@inifap.gob.mx; zetina.rigoberto@inifap.gob.mx

3 Campo Experimental Sur de Tamaulipas. Instituto Nacional de Investigaciones Agrícolas y Pecuarias (INIFAP). Km 55 carr. Tampico-Mante. Apdo. Postal C-1 Suc. Aeropuerto, 89339, Tampico, Tam., México. ascencio.guillermo@inifap.gob.mx
} 


\section{INTRODUCCIÓN}

La soya [Glycine $\max (\mathrm{L}$.$) Merr.] es un cultivo$ que tiene buena adaptación a las regiones tropicales de México (Pecina et al. 2005). Se cultiva en condiciones de riego en el sur de los estados de Sonora y Sinaloa, y bajo condiciones de temporal en el sur de Tamaulipas, el este de San Luis Potosí y las regiones del Soconusco, Chiapas y Las Huastecas, Veracruz (Esqueda et al. 1999). En México, durante 2007 se sembraron 73357 ha de soya, de las que se obtuvieron 88371,29 t de semilla (SAGARPA 2008), insuficientes para cubrir la demanda nacional de más de cuatro millones de toneladas anuales (COFEMER 2008). Esta situación señala la necesidad de incrementar la superficie sembrada con soya en el país. En pruebas experimentales y semicomerciales, la soya ha mostrado buena adaptación a las áreas tropicales de temporal del centro del estado de Veracruz (Tosquy et al. 2008), donde existen más de 100000 ha con buen potencial para su cultivo (López y Durán 1999) y diversas industrias que la utilizan como materia prima (López et al. 1994, Esqueda et al. 1997).

Para lograr que los productores del centro de Veracruz adopten este cultivo, es necesario contar con variedades y sistemas de siembra adecuados, para la obtención de altos rendimientos (Seiter et al. 2004).

La distribución inadecuada de plantas en el terreno ocasiona una ineficiente intercepción de la luz solar sobre el dosel del cultivo, y por tanto una disminución en la fotosíntesis, lo que repercute en una baja producción de semilla (Wells 1991, Savoy y Cothren 1992, Andrade et al. 2002). Una de las estrategias que se tienen para optimizar el uso de los recursos ambientales (luz, humedad, suelo y nutrimentos), contribuir a contrarrestar el problema de la sensibilidad de la soya al fotoperiodo e incrementar el rendimiento del cultivo, es el empleo de un adecuado distanciamiento entre surcos y densidad de población de plantas (Seiter et al. 2004). Así mismo, con la generación de nuevas variedades para las áreas tropicales del país, es necesario determinar la combinación óptima de distancia entre surcos y entre semillas en que las variedades expresen su máximo potencial productivo.

En la soya de temporal del norte de Veracruz y sur de Tamaulipas, se recomienda sembrar en surcos a $60 \mathrm{~cm}$, con lo que se obtiene un rendimiento hasta $25 \%$ mayor que con la práctica tradicional de surcado a 75 u 80 cm (López et al. 1994, Maldonado y Ascencio 1999). En siembras tempranas, que se establecen desde finales de junio y durante el mes de julio, se sugiere establecer poblaciones de 250000 plantas/ha para variedades de porte alto como Huasteca $200 \mathrm{y}$ de 380000 plantas/ha, para aquellas de porte bajo como Huasteca 100, mientras que en siembras tardías, establecidas en el mes de agosto, se recomiendan 300000 y 500000 plantas/haen variedades de porte alto y bajo, respectivamente (Ascencio 1999). Con estos métodos de siembra se reducen las pérdidas de semilla, ya que la altura de las vainas es adecuada para la cosecha mecánica (Maldonado y Ascencio 1999).

Considerando que el espaciamiento y la densidad de siembra óptimos de un genotipo no pueden generalizarse para todas las zonas y condiciones ambientales del país, y que en el centro del estado de Veracruz, el inicio del temporal es variable y la cantidad y distribución de la precipitación es irregular, es necesario obtener información sobre el comportamiento de variedades y métodos de siembra, en fechas tempranas, y en tardías (Grajales et al. 2006). Por lo anterior, se estableció un experimento con el objetivo de determinar el efecto de la densidad y la distancia de siembra en el comportamiento agronómico de variedades de soya.

\section{MATERIALES Y MÉTODOS}

Durante los ciclos agrícolas primavera-verano 2002 y 2003 bajo condiciones de temporal, se estableció un experimento en el lote C-7 del Campo Experimental Cotaxtla del Instituto Nacional de Investigaciones Forestales, Agrícolas y Pecuarias (INIFAP), que se localiza en el municipio de Medellín de Bravo, Veracruz, entre los $18^{\circ} 50^{\prime}$ de latitud norte y $96^{\circ} 10^{\prime}$ de longitud oeste, y una altitud de $15 \mathrm{~m}$ sobre el nivel del mar. El clima prevaleciente de la localidad es cálido subhúmedo Aw (w)(g), que corresponde a los subtipos menos húmedos de los cálidos subhúmedos (García 1987). La temperatura media anual es de $25,4^{\circ} \mathrm{C}$, con una máxima de $42,5^{\circ} \mathrm{C}$ y una mínima de $7,0^{\circ} \mathrm{C}$ y con una precipitación pluvial anual de $1400 \mathrm{~mm}$ (Díaz et al. 2006).

El suelo del lote experimental es de textura migajón-arcillosa, con pH ligeramente ácido $(6,43)$, tiene contenidos medios de materia orgánica $(2,14 \%)$, nitrógeno $(0,11 \%)$ y potasio $(160 \mathrm{ppm})$ y es extremadamente rico en fósforo (37,3 ppm) (López-Collado 1998). 
En ambos ciclos, el terreno se preparó mediante un barbecho a $30 \mathrm{~cm}$ de profundidad y dos pasos de rastra. El surcado a las distancias requeridas y la siembra del experimento se realizó el 30 de agosto en el 2002 (siembra tardía) y el 3 de julio en el 2003 (siembra temprana). Díez días después de la emergencia de la soya se raleó para dejar el número de plantas correspondiente a cada tratamiento. Inicialmente se planeó la siembra de ambos experimentos en 2002, sin embargo, debido a que la semilla sólo estuvo disponible hasta finales de agosto de ese año, el experimento de siembras tempranas se estableció en 2003. Aunque los experimentos se establecieron en años diferentes, en ambos casos se tuvieron ciclos de lluvias regulares, típicos de la zona central de Veracruz, por lo que se considera que los datos obtenidos son de utilidad para lograr los objetivos planteados.

Se evaluaron 18 tratamientos, que resultaron de la combinación de tres distanciamientos entre surcos $(40$, 60 y $75 \mathrm{~cm}$ ), tres densidades de población (200 000, 300 000 y 400000 plantas/ha) y dos variedades: Huasteca 100 y Huasteca 200. La primer variedad es de ciclo precoz, porte bajo y crecimiento semi-determinado, y la segunda de ciclo tardío, porte alto y crecimiento semideterminado (Ascencio y Maldonado 1998, Grajales 2007). Ambas variedades fueron generadas por el Programa de Soya del Campo Experimental Sur de Tamaulipas del INIFAP, para las áreas tropicales del noreste y sureste de México (Maldonado 1994). Se utilizó el diseño estadístico bloques al azar con cuatro repeticiones y diseño de tratamientos en factorial completo irregular $3 \times 3 \times 2$ con arreglo de surcos en franjas, que corresponde a los tres distanciamientos entre surcos, las tres densidades de población y las dos variedades de soya; este arreglo se utilizó para facilitar la realización de las prácticas agronómicas del cultivo. La unidad experimental constó de 12, 14 y 19 surcos de $5 \mathrm{~m}$ de longitud para las distancias de 75, 60 y $40 \mathrm{~cm}$, respectivamente. Se dejaron dos surcos de bordo al lado de cada parcela. La parcela útil tuvo una superficie de $30 \mathrm{~m}^{2}$.

Se realizaron aplicaciones del herbicida bentazón en postemergencia a $720 \mathrm{~g} /$ ha para controlar coquillo (Cyperus rotundus L.) y pelillo (C. iria L.), el 28 de septiembre de 2002 y el 25 de julio de 2003, y de fluazifop butilo a 187,5 g/ha, el 25 de septiembre de 2002, para eliminar malezas gramíneas, como zacate Johnson [Sorghum halepense (L.). Pers.] y zacate de agua [Echinochloa colona (L.) Link], como lo recomiendan Esqueda et al. (1999). También se realizaron dos pasos de cultivadora a los 15 y 25 días de nacido el cultivo en los distanciamientos a 60 y $75 \mathrm{~cm}$, y dos deshierbes con azadón en el surcado a $40 \mathrm{~cm}$. La fertilización al suelo se aplicó en banda, inmediatamente antes del segundo cultivo, y fue realizada el 30 de septiembre de 2002 y el 3 de agosto de 2003, para las siembras tardías y tempranas, respectivamente. Se utilizaron $130 \mathrm{~kg}$ de urea (46\% de N) y $87 \mathrm{~kg}$ de superfosfato de calcio triple $\left(46 \%\right.$ de $\left.\mathrm{P}_{2} \mathrm{O}_{5}\right)$, para cubrir la dosis de $60 \mathrm{~N}-40 \mathrm{P}-0 \mathrm{~K}$, sugerida para el centro de Veracruz (López et al. 1994). Durante el ciclo del cultivo se presentaron doradillas (Diabrotica balteata LeConte y Ceratomona ruficornis Olivier) y gusano peludo [Estigmenea acrea (Drury)], que se controlaron con cipermetrina a $50 \mathrm{~g} /$ ha y paratión metílico a $540 \mathrm{~g} / \mathrm{ha}$. Las aplicaciones se realizaron el 17 de septiembre y 11 de octubre de 2002, y el 24 de julio y el 15 de agosto de 2003. En el experimento establecido en fecha tardía, la cosecha se realizó el 14 de enero de 2003, mientras que la del establecido en fecha temprana, se efectuó el 25 de noviembre del mismo año.

Se tomaron datos de las siguientes variables: 1 . Días a floración media, contados a partir de la fecha de siembra hasta que el $50 \%$ de cada parcela se encontraba en floración; 2. Altura de planta en madurez fisiológica $(\mathrm{cm})$; 3. Vaina baja, medida desde la superficie del suelo hasta su parte inferior $(\mathrm{cm}) ; 4$. Número de entrenudos por planta; 5. Número de vainas por planta y 6. Rendimiento de semilla (kg/ha) ajustado al $14 \%$ de humedad. Las variables 2,3 y 4 se determinaron en cinco plantas seleccionadas al azar en la parte central de cada parcela, mientras que la variable 5 se obtuvo mediante el promedio del conteo de 15 plantas seleccionadas al azar con competencia completa. Para realizar los análisis de varianza se utilizó el paquete estadístico de la Universidad Autónoma de Nuevo León, versión 2,5 (Olivares 1994), y donde se detectó significancia, se aplicó la prueba de Tukey al 0,05 de probabilidad de error, para darle validez estadística a las medias obtenidas por cada factor en estudio y su efecto conjunto (Gómez y Gómez 1984). 


\section{RESULTADOS Y DISCUSIÓN}

\section{Siembra tardía}

En esta época de siembra, se detectaron diferencias altamente significativas en todas las variables de los efectos principales, excepto número de vainas por planta en el factor distancia entre surcos y días a floración en el factor densidad de población. También se detectaron diferencias significativas en la interacción variedad $\mathrm{x}$ distancia entre surcos en las variables altura de planta, altura de la vaina más baja y número de entrenudos por planta y en la interacción variedad $\mathrm{x}$ densidad, en altura de planta y altura de la vaina más baja. La interacción triple de estos factores sólo fue significativa para altura de la vaina más baja. Se tuvieron coeficientes de variación de entre 2,14 y 11,47\%, lo que indica buena confiabilidad de los resultados en experimentos de temporal (Reyes 1990). Los valores medios de las variables, junto con su prueba de significancia se indican en las Figuras 1 a 4 y en los Cuadros 1 a 3 .

Con los surcados a 40 y $60 \mathrm{~cm}$, la altura de planta y de vaina baja fueron significativamente mayores y se tuvieron más entrenudos por planta, que con el surcado a $75 \mathrm{~cm}$ (Figura 1). Por otra parte, los días a floración no se afectaron significativamente por los distanciamientos entre surcos. Con el distanciamiento entre surcos a $40 \mathrm{~cm}$, se obtuvo un rendimiento de semilla estadísticamente similar al de $60 \mathrm{~cm}$ y superior al de $75 \mathrm{~cm}$ (Figura 2). Sin embargo, no es recomendable surcar a $40 \mathrm{~cm}$, ya que no permite realizar las labores de cultivo y atierre. Estos resultados coinciden con los otros estudios realizados en soya de riego, cultivada en el ciclo de invierno-primavera (Tosquy et al. 2006).

En las densidades de 300000 y 400000 plantas/ ha, se tuvo mayor altura de planta y de vaina baja y número de entrenudos por planta, que con la densidad baja, aunque la altura de planta fue similar con 200000 y 300000 plantas/ha (Figura 3). Con las tres densidades de siembra la producción de vainas y el rendimiento de semilla fueron similares (Figura 4); sin embargo, no es recomendable establecer solamente 200000 plantas/ ha, debido a la poca distancia de la vaina más baja al suelo. La altura de vaina baja es una característica que debe tomarse en cuenta para la cosecha mecanizada, ya que a mayor altura de la vaina más baja al suelo, se reduce la pérdida de semilla durante la recolección (Ascencio 1999).

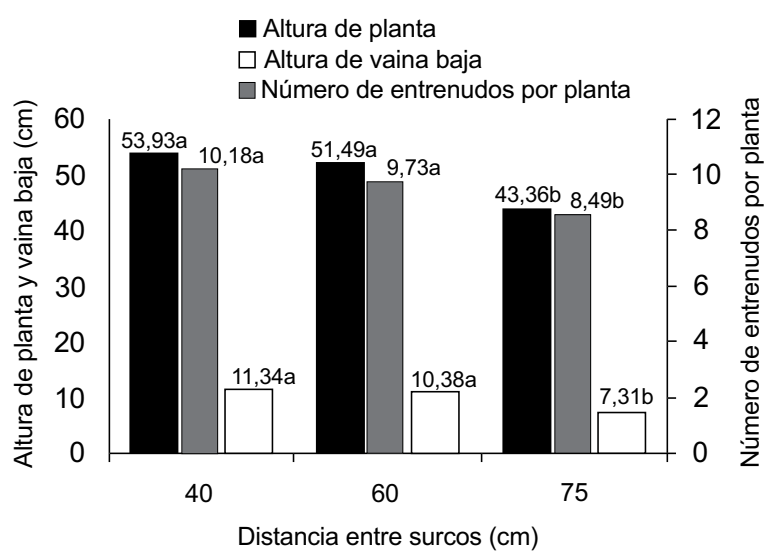

Medias con distinta letra en cada variable son estadísticamente diferentes (Tukey 0,50 )

Figura 1. Efecto de la distancia entre surcos en la altura de planta, la altura de vaina baja y en el número de entrenudos por planta de soya en siembra tardía. Medellín de Bravo, Veracruz, México. Primaveraverano 2002.

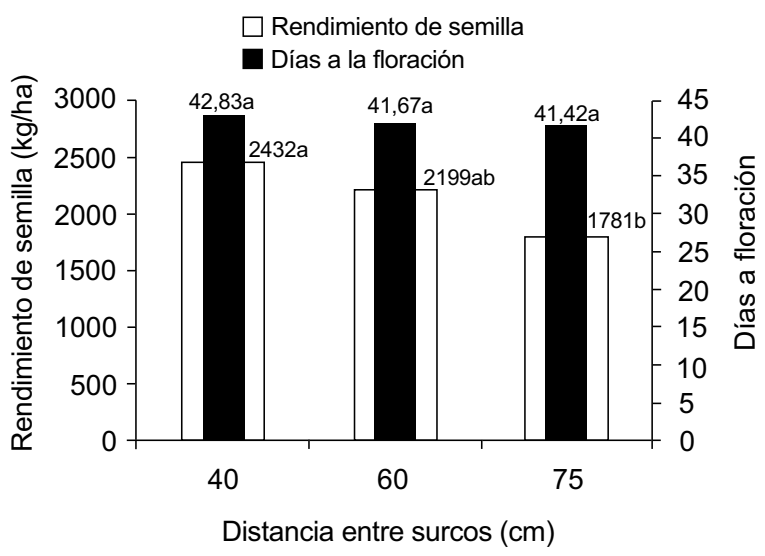

Medias con distinta letra en cada variable son estadísticamente diferentes (Tukey 0,50).

Figura 2. Efecto de la distancia entre surcos en los días a la floración y en el rendimiento de semilla de soya en siembra tardía. Medellín de Bravo, Ver., México. Primavera-verano 2002.

Aunque la altura de planta y de vaina baja y el número de entrenudos de Huasteca 100 fueron significativamente menores que los de Huasteca 200, ambos genotipos tuvieron producción de vainas y 


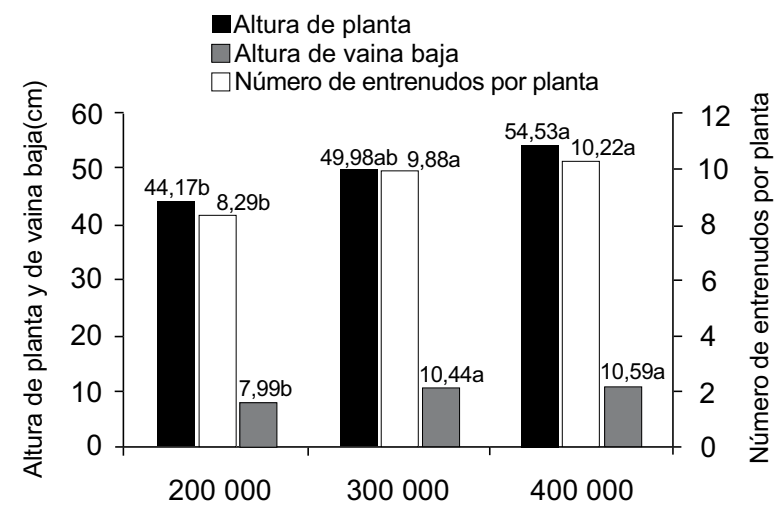

Densidad de población (plantas/ha)

Medias con distinta letra en cada variable son estadísticamente diferentes (Tukey 0,50).

Figura 3. Efecto de la densidad de población en la altura de planta, la altura de vaina baja y en el número de entrenudos por planta de soya en siembra tardía. Medellín de Bravo, Ver., México. Primavera-verano 2002.

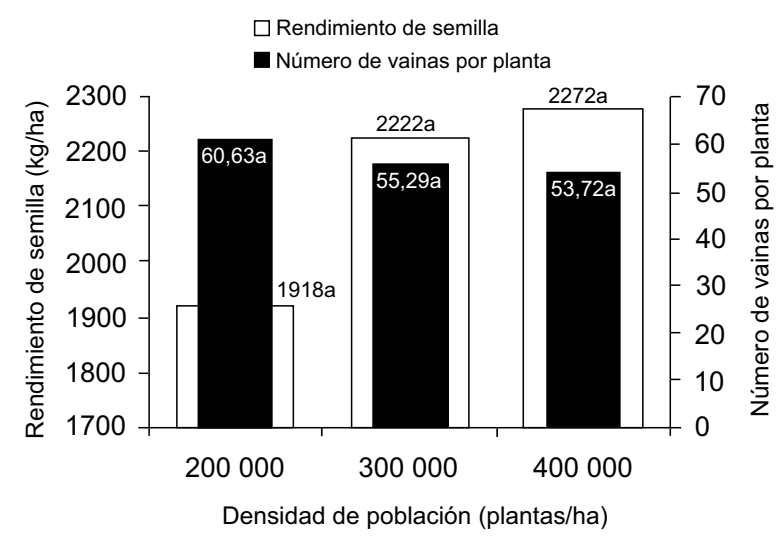

Medias con distinta letra en cada variable son estadísticamente diferentes (Tukey 0,50 ).

Figura 4. Efecto de la densidad de población en el número de vainas por planta y en el rendimiento de semilla de soya en siembra tardía. Medellín de Bravo, Ver., México. Primavera-Verano 2002.

rendimientos similares (Cuadro 1). Cabe señalar, que la variedad Huasteca 100 fue altamente sensible al fotoperíodo, y su floración se presentó 7,4 días antes que la de Huasteca 200; esta característica ha sido documentada anteriormente para las mismas variedades en el sur de Tamaulipas (Maldonado 1994). Con base en su comportamiento agronómico, esta última variedad es la más adecuada para siembras tardías de temporal de la zona central del estado de Veracruz. Es importante destacar, que durante el ciclo del cultivo, no se presentaron temperaturas mayores al límite superior óptimo de $30{ }^{\circ} \mathrm{C}$, requerido para alcanzar la máxima fotosíntesis de la soya (Díaz et al. 2007), ni hubo períodos de escasez de humedad que afectaran el rendimiento de ambos genotipos.

Con los surcados a 40 y $60 \mathrm{~cm}$, la variedad Huasteca 200 presentó una altura de planta y de vaina baja y un número de entrenudos por planta estadísticamente similares entre sí y superiores a los obtenidos con el distanciamiento a $75 \mathrm{~cm}$. A su vez, en Huasteca 100, el espaciamiento entre surcos sólo afectó significativamente la altura de vaina baja, obteniéndose también los mayores valores cuando los surcos estuvieron separados a 40 y $60 \mathrm{~cm}$ (Cuadro 2). Este mismo comportamiento se obtuvo en el sur de Tamaulipas con las variedades Huasteca 100, Huasteca 200, Santa Rosa, UFV-1, Tapachula 86 y Júpiter (Maldonado y Ascencio 1999).

En el Cuadro 3, se presenta la interacción variedad $\mathrm{x}$ densidad, donde se muestra que con $300000 \mathrm{y}$ 400000 plantas/ha, Huasteca 200 presentó una altura de planta y de vaina baja similares entre sí y superiores a las de esta misma variedad establecida con la densidad de población más baja. Similares resultados se obtuvieron para éstas y otras variedades de soya en condiciones de temporal en el estado de Chiapas, México (Grajales et al. 2006) . Este mismo comportamiento se observó en la altura de vaina baja de la variedad Huasteca 100. Los resultados indican que en siembras tardías de temporal, la variedad Huasteca 200 , se podría sembrar a una densidad de siembra de al menos 300000 plantas/ha.

En la interacción triple, la altura de vaina baja varió entre 13,9 y $16 \mathrm{~cm}$ con las combinaciones de Huasteca 200 a distancias entre surcos de 40 y $60 \mathrm{~cm}$ y densidades de 300000 y 400000 plantas/ha, las cuales fueron significativamente superiores al resto de las combinaciones (datos no presentados).

\section{Siembra temprana}

En la siembra temprana, en el factor distancia entre surcos, sólo se detectaron diferencias significativas en el número de entrenudos por planta. En la densidad de 
Cuadro 1. Comportamiento agronómico y productivo de las variedades de soya Huasteca 100 y Huasteca 200 en siembra tardía. Medellín de Bravo, Ver., México. Primavera-verano 2002.

\begin{tabular}{lcccccc}
\hline Variedad (cm) & $\begin{array}{c}\text { Altura de } \\
\text { planta }(\mathbf{c m})\end{array}$ & $\begin{array}{c}\text { Altura de vaina } \\
\text { baja }(\mathbf{c m})\end{array}$ & $\begin{array}{c}\text { \# de entrenu- } \\
\text { dos por planta }\end{array}$ & $\begin{array}{c}\text { \# de vainas por } \\
\text { planta }\end{array}$ & $\begin{array}{c}\text { Floración } \\
(\mathbf{d})\end{array}$ & $\begin{array}{c}\text { Rendimiento de } \\
\text { semilla (kg/ha) }\end{array}$ \\
\hline Huasteca 100 & $40,03 \mathrm{~b}^{*}$ & $7,61 \mathrm{~b}$ & $8,18 \mathrm{~b}$ & $53,88 \mathrm{a}$ & $38,28 \mathrm{~b}$ & $2019 \mathrm{a}$ \\
Huasteca 200 & $59,10 \mathrm{a}$ & $11,74 \mathrm{a}$ & $10,75 \mathrm{a}$ & $59,21 \mathrm{a}$ & $45,67 \mathrm{a}$ & $2256 \mathrm{a}$ \\
DMS & 5,015 & 1,116 & 0,860 & 7,307 & 1,279 & 348,7 \\
\hline
\end{tabular}

* Medias con distinta letra en una columna son estadísticamente diferentes (Tukey 0,05).

Cuadro 2. Efecto de la acción conjunta variedad x distancia entre surcos en algunas características agronómicas de la planta de soya en siembra tardía. Medellín de Bravo, Ver., México. Primaveraverano 2002.

\begin{tabular}{|c|c|c|c|c|c|c|c|}
\hline \multirow{2}{*}{$\begin{array}{l}\text { Variedad } \\
\text { Huasteca } 100\end{array}$} & \multirow{2}{*}{$\begin{array}{c}\text { Distancia entre } \\
\text { surcos }(\mathbf{c m})\end{array}$} & \multicolumn{2}{|c|}{$\begin{array}{l}\text { Altura de planta } \\
\qquad(\mathrm{cm})\end{array}$} & \multicolumn{2}{|c|}{$\begin{array}{c}\text { Altura de vaina } \\
\text { baja }(\mathrm{cm})\end{array}$} & \multicolumn{2}{|c|}{$\begin{array}{l}\text { \# de entrenudos } \\
\text { por planta }\end{array}$} \\
\hline & & 41,60 & $\mathrm{c}^{*}$ & 8,70 & $\mathrm{~b}$ & 8,58 & $\mathrm{bc}$ \\
\hline Huasteca 100 & 60 & 41,48 & $\mathrm{c}$ & 7,98 & $\mathrm{~b}$ & 8,32 & $\mathrm{bc}$ \\
\hline Huasteca 100 & 75 & 37,00 & $\mathrm{c}$ & 6,15 & $\mathrm{c}$ & 7,63 & $\mathrm{c}$ \\
\hline Huasteca 200 & 40 & 66,27 & $\mathrm{a}$ & 13,98 & $\mathrm{a}$ & 11,77 & $\mathrm{a}$ \\
\hline Huasteca 200 & 60 & 61,32 & $\mathrm{a}$ & 12,77 & $\mathrm{a}$ & 11,13 & $\mathrm{a}$ \\
\hline Huasteca 200 & 75 & 49,72 & $\mathrm{~b}$ & 8,47 & $\mathrm{~b}$ & 9,35 & $\mathrm{~b}$ \\
\hline \multicolumn{2}{|c|}{ DMS } & 7,392 & & 1,719 & & 1,267 & \\
\hline
\end{tabular}

* Medias con distinta letra en una columna son estadísticamente diferentes (Tukey 0,05).

siembra, el número de entrenudos y vainas por planta y el rendimiento de semilla mostraron diferencias significativas. A su vez, en el factor variedad, se detectaron diferencias altamente significativas en todas las variables, excepto en el número de vainas por planta. Esto último indica, que los genotipos difirieron en su comportamiento agronómico y capacidad productiva. Con respecto a las interacciones de estos factores, únicamente se encontraron diferencias significativas en variedad $\mathrm{x}$ densidad en las variables altura de planta y de vaina baja, número de entrenudos y vainas por planta y rendimiento de semilla. En el resto de las interacciones, los factores actuaron de manera independiente, por lo que los efectos simples de uno solo, fueron los mismos para todos los niveles de los otros factores dentro de una variación aleatoria, medida por el error experimental (Little y Hills 1998). Los valores promedio y prueba de significancia de las variables medidas se muestran en la Figura 5 y en los Cuadro 4 y 5.

Con el surcado a $40 \mathrm{~cm}$, las plantas de soya presentaron 14,46entrenudos, cantidadestadísticamente inferior a los 15,71, que en promedio se obtuvieron con los distanciamientos a 60 y $75 \mathrm{~cm}$. Por otra parte, con los tres espaciamientos entre surcos, el rendimiento de semilla fue ligeramente superior a $3100 \mathrm{~kg} / \mathrm{ha}$, sin haber diferencias significativas entre tratamientos (datos no presentados). Estos resultados difieren con los de Andrade et al. (2002) y Graterol y Montilla (2003), quienes obtuvieron mayor rendimiento al reducir el espaciamiento entre surcos en Argentina y Venezuela, respectivamente, situación que puede deberse a las diferentes variedades y condiciones ambientales en que se desarrolló su experimento. Sin embargo, en este caso, debido a las limitantes para 
Cuadro 3. Efecto de la acción conjunta variedad $\mathrm{x}$ densidad en altura de planta y de vaina baja de soya en siembra tardía. Medellín de Bravo, Ver., México. Primavera-verano 2002.

\begin{tabular}{lccc}
\hline Variedad & $\begin{array}{c}\text { Densidad de } \\
\text { población } \\
\text { (plantas/ha) }\end{array}$ & $\begin{array}{c}\text { Altura de } \\
\text { planta }(\mathbf{c m})\end{array}$ & $\begin{array}{c}\text { Altura de } \\
\text { vaina baja } \\
\text { (cm) }\end{array}$ \\
\hline Huasteca 100 & 200000 & $36,20 \mathrm{c}^{*}$ & $6,33 \mathrm{c}$ \\
Huasteca 100 & 300000 & $40,95 \mathrm{c}$ & $8,27 \mathrm{~b}$ \\
Huasteca 100 & 400000 & $42,93 \mathrm{c}$ & $8,23 \mathrm{~b}$ \\
Huasteca 200 & 200000 & $52,15 \mathrm{~b}$ & $9,65 \mathrm{~b}$ \\
Huasteca 200 & 300000 & $59,02 \mathrm{ab}$ & $12,62 \mathrm{a}$ \\
Huasteca 200 & 400000 & $66,13 \mathrm{a}$ & $12,95 \mathrm{a}$ \\
\hline DMS & & 7,392 & 1,719 \\
\hline
\end{tabular}

* Medias con distinta letra en una columna son estadísticamente diferentes (Tukey 0,05).

efectuar las labores mecánicas antes mencionadas, para las siembras tempranas de soya de temporal es preferible utilizar las distancias de 60 y $75 \mathrm{~cm}$.

Con la densidad de población más baja, las plantas de soya tuvieron un número significativamente mayor de entrenudos que con la densidad más alta y produjeron, en promedio, más vainas por planta que cuando se establecieron a 300000 y 400000 plantas/ha. Sin embargo, los mayores rendimientos de semilla $(\mathrm{p}<0,05)$ se obtuvieron con estas dos últimas poblaciones de plantas, aunque el rendimiento obtenido con 200000 plantas/ha fue estadísticamente semejante al de la densidad mayor (Figura 5). Esto pone de manifiesto que un mayor número de entrenudos y vainas por planta no necesariamente se traduce en mayores rendimientos de semilla, debido a que en este caso la densidad de

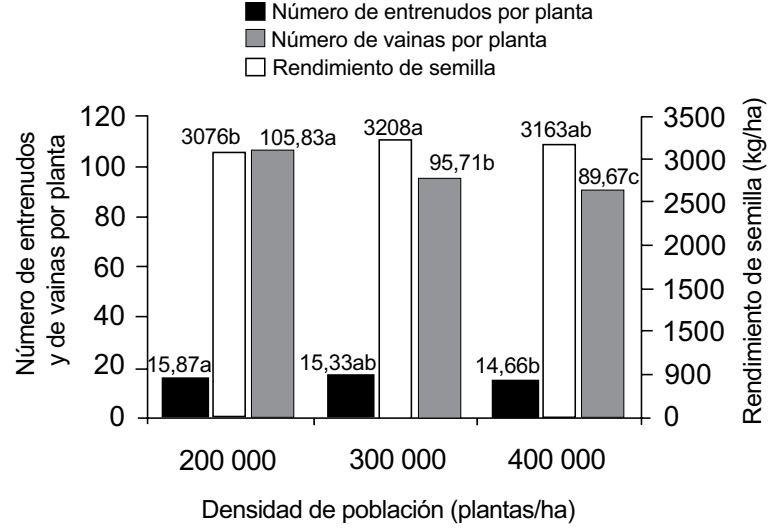

Medias con distinta letra en cada variable son estadísticamente diferentes (Tukey 0,50).

Figura 5. Efecto de la densidad de población en el número de entrenudos y vainas por planta y en el rendimiento de semilla de soya en siembra temprana. Medellín de Bravo, Ver., México. Primavera-verano 2003.

siembra fue un factor más determinante que estas dos variables. Lo anterior coincide con lo señalado por Parvez et al. (1989) y Holshouser y Whittaker (2002), quienes encontraron que el rendimiento de semilla es afectado por el método y la densidad de población. Por lo anterior, para obtener los rendimientos más altos en siembras tempranas de temporal, es suficiente establecer una población de 300000 plantas/ha.

La variedad Huasteca 200 tuvo mayor altura de planta y de vaina baja y presentó más entrenudos que Huasteca 100, además de que su floración fue significativamente más tardía (Cuadro 4). Sin embargo, el rendimiento de esta última variedad fue significativamente superior. Aunque la altura de vaina baja

Cuadro 4. Comportamiento agronómico y productivo de las variedades de soya Huasteca 100 y Huasteca 200 en siembra temprana. Medellín de Bravo, Ver., México. Primavera-verano 2003.

\begin{tabular}{lccccc}
\hline Variedad & $\begin{array}{c}\text { Altura de planta } \\
(\mathbf{c m})\end{array}$ & $\begin{array}{c}\text { Altura de vaina } \\
\text { baja }(\mathbf{c m})\end{array}$ & $\begin{array}{c}\text { \# de entrenudos } \\
\text { por planta }\end{array}$ & Floración (d) & $\begin{array}{c}\text { Rendimiento de } \\
\text { semilla (kg/ha) }\end{array}$ \\
\hline Huasteca 100 & $96,47 \mathrm{~b}^{*}$ & $13,64 \mathrm{~b}$ & $14,53 \mathrm{~b}$ & $47,25 \mathrm{~b}$ & $3227 \mathrm{a}$ \\
Huasteca 200 & $107,22 \mathrm{a}$ & $16,00 \mathrm{a}$ & $16,05 \mathrm{a}$ & $53,42 \mathrm{a}$ & $3071 \mathrm{~b}$ \\
DMS & 2,191 & 0,824 & 0,585 & 0,475 & 83,1 \\
\hline
\end{tabular}

* Medias con distinta letra en una columna son estadísticamente diferentes (Tukey 0,05). 
Cuadro 5. Efecto de la acción conjunta variedad x densidad en características agronómicas y rendimiento de soya en siembra temprana. Medellín de Bravo, Ver., México. Primavera-verano 2003.

\begin{tabular}{lcccccc}
\hline Variedad & $\begin{array}{c}\text { Densidad } \\
\text { (plantas/ha) }\end{array}$ & $\begin{array}{c}\text { Altura de } \\
\text { planta }(\mathbf{c m})\end{array}$ & $\begin{array}{c}\text { Altura de vaina } \\
\text { baja }(\mathbf{c m})\end{array}$ & $\begin{array}{c}\text { \# de entrenudos } \\
\text { por planta }\end{array}$ & $\begin{array}{c}\text { \# de vainas por } \\
\text { planta }\end{array}$ & $\begin{array}{c}\text { Rendimiento de } \\
\text { semilla (kg/ha) }\end{array}$ \\
\hline Huasteca 100 & 200000 & $95,08 \mathrm{c} *$ & $12,83 \mathrm{c}$ & $13,67 \mathrm{c}$ & $101,92 \mathrm{~b}$ & $2925 \mathrm{~d}$ \\
Huasteca 100 & 300000 & $98,83 \mathrm{c}$ & $14,00 \mathrm{bc}$ & $15,25 \mathrm{~b}$ & $96,33 \mathrm{bc}$ & $3454 \mathrm{a}$ \\
Huasteca 100 & 400000 & $95,50 \mathrm{c}$ & $14,08 \mathrm{bc}$ & $14,67 \mathrm{bc}$ & $90,00 \mathrm{~d}$ & $3301 \mathrm{ab}$ \\
Huasteca 200 & 200000 & $111,17 \mathrm{a}$ & $17,00 \mathrm{a}$ & $18,08 \mathrm{a}$ & $109,75 \mathrm{a}$ & $3228 \mathrm{bc}$ \\
Huasteca 200 & 300000 & $105,00 \mathrm{~b}$ & $15,41 \mathrm{ab}$ & $15,42 \mathrm{~b}$ & $95,08 \mathrm{~cd}$ & $2961 \mathrm{~d}$ \\
Huasteca 200 & 400000 & $105,50 \mathrm{~b}$ & $15,58 \mathrm{ab}$ & $14,67 \mathrm{bc}$ & $89,33 \mathrm{~d}$ & $3026 \mathrm{~cd}$ \\
\hline \multicolumn{2}{c}{ DMS } & 5,593 & 2,104 & 1,495 & 6,163 & 212,2 \\
\hline
\end{tabular}

* Medias con distinta letra en una columna son estadísticamente diferentes (Tukey 0,05).

de Huasteca 100 fue menor a la de Huasteca 200, no representa una limitante para la cosecha mecanizada en siembras tempranas de temporal, ya que su altura fue de promedio superior a $13 \mathrm{~cm}$ (Maldonado 1994). Lo anterior es importante, ya que generalmente las variedades de ciclo corto, producen vainas muy bajas que no son cosechadas, y reducen el rendimiento de semilla (Grajales et al. 2006). Al igual que en la siembra tardía, las condiciones de temperatura y precipitación pluvial en este ciclo de siembra fueron adecuadas para que las variedades expresaran su mayor potencial productivo.

En este estudio, el rendimiento promedio de los dos genotipos, fue muy superior al que normalmente se obtiene en las siembras comerciales de temporal de la zona norte del estado (Tosquy et al. 2006). Lo anterior indica que en la parte central del estado de Veracruz, durante el ciclo primavera-verano se tiene un buen potencial para producir soya de temporal en fechas tempranas. En esas condiciones, la variedad Huasteca 100, muestra mayor potencial de rendimiento que Huasteca 200, y buen comportamiento agronómico.

La interacción variedad $\mathrm{x}$ densidad (Cuadro 5) mostró que al establecer Huasteca 200 a 200000 plantas/ha, las plantas tuvieron una altura significativamente mayor, formaron más entrenudos y produjeron en promedio más vainas por planta que en el resto de las combinaciones de estos factores. Con esta combinación también se obtuvo la mayor altura de vaina baja, la cual fue estadísticamente semejante a la obtenida con la misma variedad sembrada a 300000 y 400000 plantas/ha, y superior a la de Huasteca 100 en cualquiera de las densidades. El mayor rendimiento de semilla de la combinación variedad x densidad se obtuvo con Huasteca 100 a 300000 plantas/ha, el cual fue estadísticamente similar al obtenido con la misma variedad a 400000 plantas/ha y superior al de las otras combinaciones.

La diferente respuesta de las variedades a las densidades de población, se debe a que estos materiales son genotípica y fenotípicamente diferentes, lo que las hace interaccionar de manera distinta con el ambiente (Maldonado 1994). En este sentido, por ser un genotipo de porte más bajo, Huasteca 100 puede establecerse a una mayor densidad de población que Huasteca 200, la cual tiene mejor desarrollo y comportamiento agronómico a baja densidad.

\section{LITERATURA CITADA}

Andrade, FH; Calviño P; Cirilo, A; Barbieri, P. 2002. Yield responses to narrow rows depend on increased radiation interception. Agronomy Journal 94:975-980.

Ascencio, LG; Maldonado, MN. 1998. Crecimiento y desarrollo de la soya en el sur de Tamaulipas, México. Agricultura Técnica en México 24(2):99-110.

Ascencio, L. G. 1999. Métodos de siembra en soya. In: Cruz, BJA; Maldonado, MN; Hinojosa, RI; Elizondo, BJ. eds. Memoria del Curso-Taller de Capacitación Técnica en el Cultivo de Soya. Publicación Especial Núm. 1. 
SAGAR. INIFAP. CIRGOC. Campo Experimental Cotaxtla. Medellín de Bravo, Veracruz, México. p. 9-14.

COFEMER (Comisión Federal de Mejoría Regulatoria). 2008. Problemática del abasto en condiciones competitivas internacionalmente (en línea). Consultado 21 nov. 2008. Disponible en http://www.cofemermir.gob.mx/uploadtests/ 4980.62.59.1.PROBLEM $\%$ C3\%81TICA\%20DEL \%20CONSUMO\%20DE\% 20PASTA\%20DE\%20SOYA2.doc

Díaz, PG; Ruíz, CJA; Cano, GMA; Serrano, AV; Medina, GG. 2006. Estadísticas climatológicas básicas del estado de Veracruz (período 1961-2003). Libro Técnico Núm. 13. SAGARPA. INIFAP. CIRGOC. Campo Experimental Cotaxtla. Veracruz, México. 292 p.

Díaz, PG; Medina, GG; Ruíz, CJA; Serrano, AV. 2007. Potencial productivo del cultivo de soya (Glycine max (L.) Merr.) en México. Ciclo primavera-verano. Delimitación en sistemas de información geográfica. Publicación Técnica Núm. 2. SAGARPA. INIFAP. CIRGOC. Campo Experimental Cotaxtla. Veracruz, México. 67 p.

Esqueda, VA; Durán, PA; López, SE; Cano, RO. 1997. Efecto de la competencia y de la época de limpia de la maleza en soya Glycine max (L.) Merr. de temporal en el centro de Veracruz. Agricultura Técnica en México 23(1):27-40.

Esqueda, VA; López, GVO; López, SE. 1999. Control de maleza en soya (Glycine max L. Merr.) de temporal con herbicidas. Agricultura Técnica en México 25(1):41-51.

García, E. 1987. Modificaciones al sistema de clasificación climática de Köppen (para adaptarlo a las condiciones de la República Mexicana). 4 ed. Universidad Nacional Autónoma de México. México, D.F. 130 p.

Gomez, KA; Gomez, AA. 1984. Statistical procedures for agricultural research. 2 ed. Wiley, New York, USA. $680 \mathrm{p}$.

Grajales, SM; Alonso, BM; Aguirre, MJF; Fraire, VG; Reza, AR; De León, EF. 2006. El cultivo de la soya Glycine $\max$ L. Merril. Libro Técnico No. 3. INIFAP. CIRPAS. Campo Experimental Rosario Izapa. Tuxtla Chico, Chis., México. 185 p.

Grajales, SM. 2007. Guía para cultivar soya de temporal en Chiapas. Folleto para Productores Núm. 4. INIFAP. CIRPAS. Campo Experimental Rosario Izapa. Tuxtla Chico, Chis., México. 34 p.

Graterol, Y; Montilla, D. 2003. Efecto de distancias de siembra y poblaciones sobre el comportamiento de dos cultivares de soya de crecimiento indeterminado. Bioagro 15(3):183-192.
Holshouser, DL; Whittaker, JP. 2002. Plant population and row-spacing effects on early soybean production systems in the Mid-Atlantic USA. Agronomy Journal 94:603-611.

Little, MT; Hills, FJ. 1998. Métodos estadísticos para la investigación en la agricultura. 2 ed. Trillas. México, D. F. 270 p.

López, SE; Durán, PA; Becerra, LEN; Esqueda, EVA; Espinosa, De Los MJ; Cano, RO; Cumpián, GJ. 1994. Manual para cultivar soya de temporal en el centro de Veracruz. Folleto para Productores No. 8. SAGARPA. INIFAP. CIRGOC. Campo Experimental Cotaxtla. Veracruz, México. 24 p.

López-Collado, CJ. 1998. Interpretación de los resultados de los análisis químicos de suelos agrícolas. Colegio de Postgraduados. Campus Veracruz. Manlio Fabio Altamirano, Veracruz, México. 45 p.

López, GV; Durán, PA. 1999. Potencial productivo de soya para el estado de Veracruz. In: Cruz, BJA; Maldonado, MN; Hinojosa, RI; Elizondo, BJ. eds. Memoria del Curso-Taller de Capacitación Técnica en el Cultivo de Soya. Publicación Especial Núm. 1. SAGAR. INIFAP. CIRGOC. Campo Experimental Cotaxtla. Medellín de Bravo, Veracruz, México. p. 41-51.

Maldonado, MN. 1994. Huasteca 100 y Huasteca 200, nuevas variedades de soya para el sur de Tamaulipas. Folleto para Productores No. 9. SARH. INIFAP. CIRNE. Campo Experimental Sur de Tamaulipas. Tamaulipas, México. 21 p.

Maldonado, MN; Ascencio, LG. 1999. Siembra de soya en surco angosto. Desplegable para productores \#. 4. SAGAR. INIFAP. CIRNE. Campo Experimental Sur de Tamaulipas. Est. Cuauhtémoc, Tam., México.

Olivares, SE. 1994. Paquete estadístico de diseños experimentales (programa de cómputo) versión 2.5. Facultad de Agronomía de la Universidad Autónoma de Nuevo León. Marín, N.L., México.

Parvez, AQ; Gardner, FP; Boote, KJ. 1989. Determinate and indeterminate type soybean cultivar responses to pattern density and planting date. Crop Science 29:150-157.

Pecina, QV; Maldonado, HL; Maldonado, MN; Simpson, J; Martínez, VO; Gil, V. K. C. 2005. Diversidad genética en soya del trópico húmedo de México determinada con marcadores AFLP. Revista Fitotecnia Mexicana 28(1):63-69.

Reyes, CP. 1990. Diseño de experimentos aplicados. 3 ed. Trillas. México, D.F. 348 p.

SAGARPA (Secretaría de Agricultura, Ganadería, Desarrollo Rural, Pesca y Alimentación). 2008. Anuario estadístico 
de la producción agrícola. Servicio de Información Agroalimentaria y Pesquera (SIAP). Avances de siembras y cosechas (en línea). Consultado 20 nov. 2008. Disponible en http://www.siap.sagarpa.gob.mx

Savoy, BR; Cothren, JT. 1992. Soybean biomass accumulation and leaf area index in early-season production environments. Agronomy Journal 84:956-959.

Seiter, S; Altemose, CE; Davis, MH. 2004. Forage soybean yield and quality responses to plant density and row distance. Agronomy Journal 96:966-970.

Tosquy, VOH; Esqueda, EVA; Durán, PA. 2006. Sistemas de siembra para soya de invierno en Veracruz, México. 2006. Agronomía Mesoamericana 17(1):63-69.
Tosquy, VOH; Esqueda, EVA; Durán, PA; Jácome, MSM; Ugalde, AFJ. 2008. Sistemas de siembra para soya de temporal en el centro del estado de Veracruz. In: Barradas, LHV; Esqueda, EVA; Tosquy, VOH; Vinay, VJC; Hernández, BA; Domínguez, MB. et al. eds. Avances en la Investigación Agrícola, Pecuaria, Forestal y Acuícola en el Trópico Mexicano 2008. Libro Científico No. 5. INIFAP. UV. CP. UACH. ITUG. ITBOCA. UNAM. Veracruz, México. p. 71-78.

Wells, R. 1991. Soybean growth response to plant density: relationships among canopy photosynthesis, leaf area, and light interception. Crop Science 31:755-761. 